\title{
Estimating the circulation from hydrography and satellite altimetry in the Southern Ocean: limitations imposed by the current geoid models
}

\author{
Martin Losch*, Jens Schröter \\ Alfred-Wegener-Institut für Polar- und Meeresforschung, Postfach 120161, Haus F, Bremerhaven 27515, Germany
}

Received 17 June 2003; received in revised form 8 October 2003; accepted 24 February 2004

\begin{abstract}
Sea-surface height data from satellite altimetry provide a very powerful means of determining the general ocean circulation. For oceanographic studies in which the absolute sea-surface height is required, one has to use the equipotential height of a geoid model as a reference surface. Earlier studies have shown that with recent geoid models, this reference surface is not known to an accuracy sufficient for ocean state estimation. It is demonstrated with an analysis of a hydrographic section between Australia and Antarctica that the combination of altimetry data and the geoid height of a state-of-the-art geoid model is not only inaccurate, but also inconsistent with a hydrographic estimate of the flow field. The conclusion is drawn that this is so because the formal errors of the geoid model underestimate its true errors.
\end{abstract}

(C) 2004 Elsevier Ltd. All rights reserved.

Keywords: Ocean circulation; Hydrography; Satellite altimetry; Geoid; Inverse methods

\section{Introduction}

Estimating the state of the ocean relies heavily on the data available. In spite of the enormous efforts that have been undertaken in the past, the ocean-because of its vast dimensions compared to the size and speed of research vessels - must still be considered as under-sampled. The advent of air- and space-borne measurement techniques

\footnotetext{
*Corresponding author. Tel.: + 49-471-4831-1872; fax: +49471-4831-1797.

E-mail address: mlosch@awi-bremerhaven.de (M. Losch).
}

opened a rich source of information with high spatial and temporal resolution. Of these new types of data, sea-surface height measurements by satellite-based altimetry appear the most successful for estimating the flow field, because the sea-surface height directly reflects the three-dimensional, largescale circulation (Wunsch and Stammer, 1998).

Many studies are now devoted to the use of satellite altimetry data, most of which explore time-dependent problems and sea-surface height variability exploiting the high-precision measurements obtained by the TOPEX/POSEIDON-mission. Before using the altimetry data for studies 
that require an estimate of the absolute sea-surface height, one has to subtract an equipotential surface, the geoid undulation or geoid height, from the measurements in order to obtain the physically relevant surface elevation (Wunsch and Stammer, 1998). Numerous studies have demonstrated how altimetry data can be successfully combined with hydrographic data and physical conservation laws to give improved estimates of the ocean circulation (e.g., Martel and Wunsch, 1993; Ganachaud et al., 1997; LeGrand, 2001; Wenzel et al., 2001; Schröter et al., 2002; Losch et al., 2002a). However, many of these studies used artificial scenarios instead of the actual altimetry data, since up to now the geoid height, which is calculated from a geoid model, has been found to be insufficiently accurate to have a significant impact on improving estimates of the ocean circulation (Ganachaud et al., 1997). Even the state-of-the-art geoid model Earth Gravitational Model, 1996 (EGM96) (Lemoine et al., 1997) can only be found rarely in applications in ocean science, for example, in Stammer et al. (2002). As an explanation for this in accuracy, we put forward the hypothesis that present day geoid models in general, and the EGM96 as the latest prior to dedicated gravity space missions in particular, have error estimates that make them inconsistent with our present understanding of oceanic motion. We test this hypothesis in a region south of Australia, where due to the general sparseness of data in the Southern Ocean, we expect the impact of new data to be most important.

Earlier studies support our point of view. Stammer and Wunsch (1994) and Rapp et al. (1996) have shown for predecessors of the EGM96 that even at very long wavelengths above $2500 \mathrm{~km}$, for which the geoid is well known, the difference between sea-surface height estimates by general circulation models, hydrographic dynamic height calculations, and satellite altimetry exceeds $10 \mathrm{~cm}$ in the root-mean-square. Wunsch (1993) and Martel and Wunsch (1993) demonstrated by means of an inverse model for the North Atlantic that satellite altimetry relative to a geoid surface overestimates sea-surface height gradients. To these authors the error estimates of the geoid seemed too small by a factor of two. Furthermore in a more recent study, LeGrand (2001) remarked in passing that the formal errors of the EGM96 geoid height are too small to be consistent with sea-surface height data and his inverse ocean model, but did not make a quantitative statement. In a subsequent paper, LeGrand et al. (2003) revised the previous statement and presented an inverse model solution that combined the EGM96 geoid height with satellite altimetry and climatological hydrography. The authors attributed their success to the use of a revised prior error covariance for the geoid height. In the earlier paper, LeGrand (2001) had used a simplified form for his error estimates.

To our knowledge, Stammer et al. (2002) is the only publication in which the possibility of an underestimated EGM96 geoid error was discussed in some detail in an oceanographic modeling context. The authors found the differences between the geoid height implied by their model and the EGM96 (which had been used in their assimilation experiment) too large to be consistent with the prior error statistics. They attributed these residuals to "geoid errors in excess of those formally estimated for EGM96" and provided some evidence to support their hypothesis.

However in Stammer et al. (2002), the analysis is necessarily incomplete: the enormous size of their model prohibited a posterior error analysis. Therefore, Stammer et al. (2002) could provide neither a prior nor a posterior consistency check so that it may still be possible that their solution is consistent with EGM96. We choose a computationally less expensive model to demonstrate the inconsistency between hydrography and satellite altimetry. This allows us to perform an estimation that is complete with error calculations. In addition to the localized discrepancies detected by Stammer et al. (2002), and in contrast to LeGrand et al. (2003), we show that even on large scales, where the geoid is supposedly known rather well, inconsistencies are found.

Ganachaud et al. (1997) reported that TOPEX/ POSEIDON altimetry in combination with the JGM-3 geoid model, a predecessor of the the EGM96, is consistent with an independent circulation estimate by a global box inverse model. On 
the other hand, the geoid model errors they used were so large, that including the sea-surface height data in the inversion did not improve the solution, i.e., it did not reduce the posterior error estimates significantly. In contrast to Ganachaud et al.'s conclusions with the JGM-3, we illustrate and discuss that in the context of an inverse section model, the EGM96, which has much smaller formal errors than the JGM-3, and hydrography are not consistent. This result should discourage oceanographers from naively constraining ocean models with absolute sea-surface topographies relative to an EGM96 geoid height. The difficulties with EGM96 will be obsolete once data from ongoing (and planned) high-precision gravity missions have arrived and been evaluated, with a full account of the error covariances. However, until then geoid models such as the EGM96 provide the best available geoid height estimates.

Our general procedure for this note can be outlined as follows. The model is described in Section 2. In Section 3, we briefly discuss an estimate of the mean flow field south of Australia derived from hydrography. We compare this estimate, which is consistent with other independent estimates, to the available sea-surface height data in Section 4. In order to overcome the inconsistencies between hydrographic estimate and altimetric measurement, we have to revise the prior error estimates of the inverse model. Alternatively we may revise the prior error estimate of the geoid model, or both. We will show that revising only the inverse model's prior errors does not lead to satisfactory results and that it is necessary to increase the geoid error estimates. This is the purpose of our presentation. After increasing the estimated error of the inverse model by a factor of 5 it is possible to combine hydrography and altimetry. A new estimate is found that is statistically consistent with our revised a priori assumptions. This new solution is then compared to the independent estimates of the flow south of Australia. It will be shown that the new solution is unrealistic and must be rejected. This implies that it is inadequate to raise the error level of the ocean model substantially. Instead the errors of the geoid model need revision.

\section{Inverse model}

The model used here is based on the non-linear inverse section model introduced by Nechaev and Yaremchuk (1995). For the purpose of this note it suffices to state that this model calculates geostrophic velocities from hydrographic section data via the UNESCO equation of state of sea-water and thermal wind from first guesses of temperature and salinity fields. These first guesses are taken directly from the representation of the hydrographic measurements on the model grid. The model estimates are compared to data in a cost function; by an iterative algorithm, using the adjoint technique, new estimates of the model variables are found that minimize the cost function. The most important unknowns of this problem are the so-called reference velocities of the classical dynamic method, which in our case are estimated a priori to be zero at the bottom (S. Rintoul, pers. comm., 1999).

The model is different from "traditional" box inverse models (e.g., Wunsch, 1978) in that it includes temperature and salinity as control parameters that are estimated in the inversion process. In this sense, it is more flexible and can account for errors in the hydrographic fields explicitly. Furthermore, we seek smooth solutions by constraining the second derivatives of the tracer fields. A detailed description of the model and its performance can be found in Losch et al. (2002a). There, it is illustrated how the model can estimate a mean (time-independent) circulation from synoptic hydrography and mean sea-surface height data in a twin experiment study.

The solution to the inverse problem is defined by specifying errors and their correlations for the individual data and model parameters a priori. The inverses of these prior errors are used as weights for the individual cost function terms. The prior error estimates and the construction of the weights have been described previously by, for example, Yaremchuk et al. (2001) and Losch et al. (2002a). For the sake of brevity, we describe only the weights of the data sets of immediate interest here. The weights for sea-surface height data are discussed in Section 4 and the appendix. The prior error covariance for the hydrography is the sum of 
a diagonal noise term of the order of $0.5^{\circ} \mathrm{C}$ for temperature and 0.02 for salinity and a nondiagonal correlation matrix. Correlations in the vertical and between temperature and salinity are estimated from the hydrography for each hydrographic station, while horizontal correlations are neglected. The resulting covariances have diagonal terms that correspond to errors of the order of $5^{\circ} \mathrm{C}$ for temperature and 0.5 for salinity. The large prior errors allow the hydrographic fields to mutually adjust according to the prior correlation estimate. The error covariance matrices for each station are inverted to obtain the weight matrices.

The final estimate of the inverse model satisfies geostrophy and the equation of state exactly. To this extent, the model is very similar to the classical dynamic method. But our model yields estimates of both the flow field and its associated errors. The error estimate is calculated by inverting the Hessian matrix of second derivatives of the cost function to obtain an error covariance matrix for the independent parameters. The errors of the dependent variables, for example transports across the section, can then be found from their linearized dependence on independent parameters, for example, the reference velocities (e.g., Thacker, 1989).

\section{Hydrographic estimate of the flow field south of Australia}

We analyze the temperature and salinity measurements of the January 1994 cruise along of the World Ocean Circulation Experiment Repeat Section SR3 (Rosenberg et al., 1995). We choose this particular data set because, of all available cruises, it has the best set of tracer measurements. We also considered using the mean hydrography of all available cruises, but decided that this would introduce more inconsistencies due to different station locations, etc., than it would remove. Also the inverse model was designed to estimate the mean flow from synoptic data (Losch et al., 2002a). The SR3-section runs from the southern tip of Tasmania across the Southern Ocean along approximately $140-145^{\circ} \mathrm{W}$ to the Terre Adélie shelf off the Antarctic continent (Fig. 1). Tem-

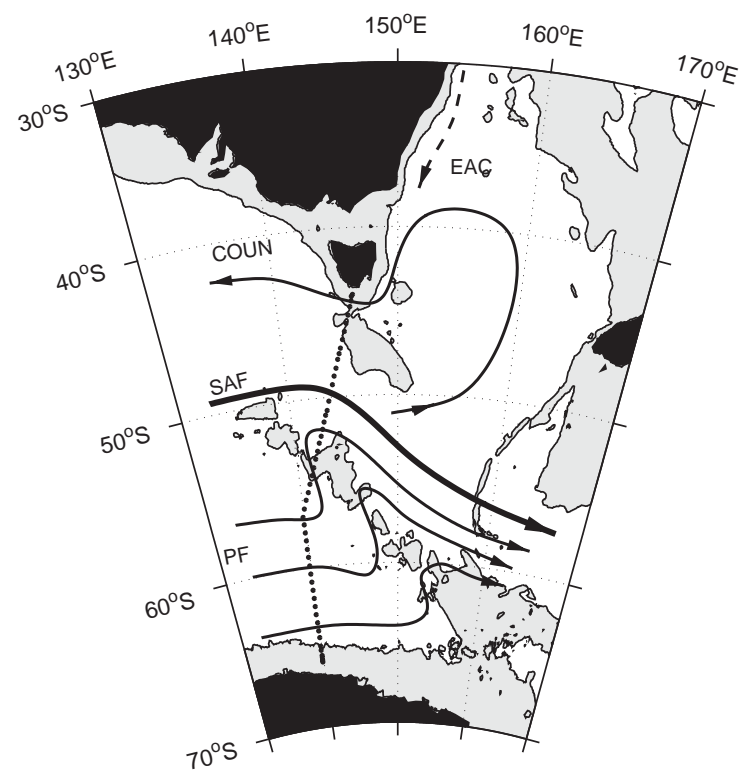

Fig. 1. Location of the Southern Ocean Repeat section SR3 between Tasmania and Antarctica with currents systems after Rintoul et al. (2001). SAF stands for Subantarctic Font, EAC denotes the East Australian Current, and PF the Polar Front(s). Areas with water depths above $3000 \mathrm{~m}$ are shaded.

perature and salinity measurements are interpolated onto 34 standard depths, which are 10-100 m apart above $1500 \mathrm{~m}$ water depth and $250 \mathrm{~m}$ apart below that. The deepest measurement is at $4500 \mathrm{~m}$. For a more complete description of the hydrography and the flow field along SR3 the reader is referred to Yaremchuk et al. (2001), who used a model setup that is similar to ours, and to Rintoul and Sokolov (2001) and Rintoul et al. (2001).

For our discussion we focus on the velocities and sea-surface height. The estimates of both temperature and salinity fields are not shown. In spite of the relatively large prior error estimates (i.e., small weights in the cost function), the large scale structure of the hydrographic fields differs only marginally from that of the data after the inversion, as shown by Yaremchuk et al. (2001). The fields are, however, smoother than the data, thereby diminishing small scale features. The rootmean-square difference of our temperature and salinity estimate with a hydrographic atlas, such as the WOCE-SAC atlas (Gouretski and Jancke, 1998), which is necessarily a mean estimate, 
decreases by approximately $20 \%$, thereby justifying our choice of weights.

The flow field of the mean estimate from hydrographic data alone is shown in the top panel of Fig. 2. Although this estimate does not include the three measurements from current moorings used in Yaremchuk et al. (2001), it still resembles their solution for the particular cruise (January 1994). There is a narrow eastward boundary current, which carries $1 \pm 1 \mathrm{~Sv} \quad(1 \mathrm{~Sv}=$ $\left.10^{6} \mathrm{~m}^{3} \mathrm{~s}^{-1}\right)$, and a westward counter current south of the Tasman continental rise, which carries $16 \pm 7 \mathrm{~Sv}$. The core of the Antarctic Circumpolar Current (ACC) in the Subantarctic Front between
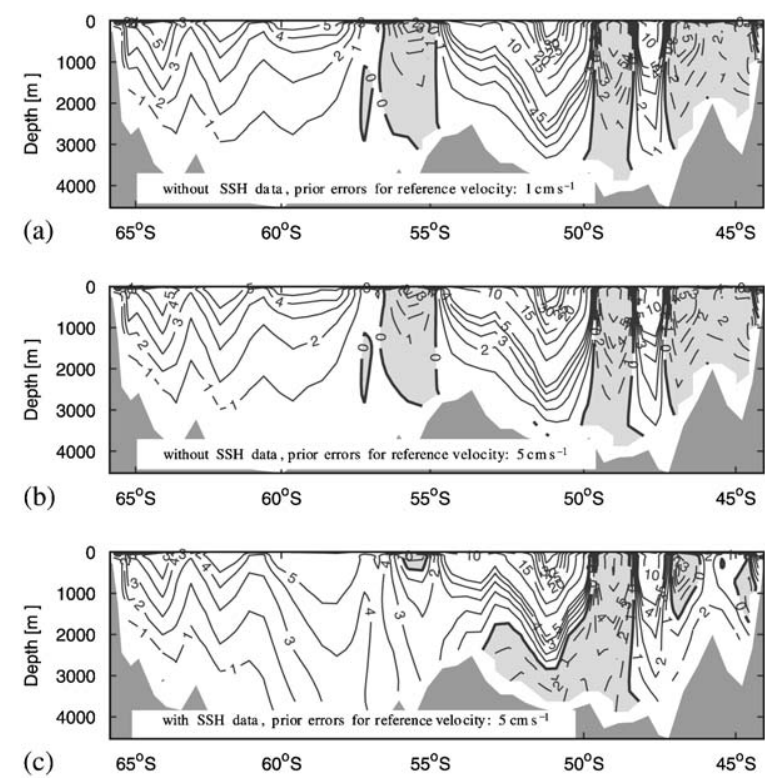

Fig. 2. Velocity estimates normal to section SR3 for three experiments: (a) estimate from hydrography alone with a small prior error for the reference velocity of $1 \mathrm{~cm} \mathrm{~s}^{-1}$; this estimate is consistent with previous estimates of transports through the section; (b) same as (a), but the prior error for the reference velocity has been increased to $5 \mathrm{~cm} \mathrm{~s}^{-1}$ to make the model's seasurface height estimate consistent with the altimetry data; (c) estimate after combination of hydrography and sea-surface height data with a revised prior reference velocity error of $5 \mathrm{~cm} \mathrm{~s}^{-1}$. Contour interval is $5 \mathrm{~cm} \mathrm{~s}^{-1}$ for current speeds greater than 5 and $1 \mathrm{~cm} \mathrm{~s}^{-1}$ for speeds smaller than $5 \mathrm{~cm} \mathrm{~s}^{-1}$. Shaded areas with dashed contours denote westward velocity. The significant undercurrent below the Subantarctic Front at $51^{\circ} \mathrm{S}$ in (c) is in contradiction to the latest estimate by Rintoul and Sokolov (2001). $50^{\circ} \mathrm{S}$ and $55^{\circ} \mathrm{S}$ has a transport of $115 \pm 6 \mathrm{~Sv}$. South of $57^{\circ} \mathrm{S}$, there is a broad drift current of $60 \pm 9 \mathrm{~Sv}$. Between $55^{\circ} \mathrm{S}$ and $57^{\circ} \mathrm{S}, 6 \pm 4 \mathrm{~Sv}$ of this drift current turn, according to Rintoul and Sokolov (2001), northward and even westward before they join with the core flow of the ACC.

We estimate smaller transport errors than Yaremchuk et al. (2001) because we chose a small prior error for the reference velocities of only $1 \mathrm{~cm} \mathrm{~s}^{-1}$. In other words, the reference velocity of the final state is allowed to deviate from the initial guess by one standard deviation of $1 \mathrm{~cm} \mathrm{~s}^{-1}$. This choice is justified since our estimate of the mean total transport through the SR3-section of $152 \pm 13 \mathrm{~Sv}$ agrees with previous estimates of the order $150 \pm 15 \mathrm{~Sv}$ by, for example, Macdonald (1998), Sloyan and Rintoul (2000), Ganachaud and Wunsch (2000), and Rintoul and Sokolov (2001). However, because the bottom velocities are nearly zero, the baroclinic structure of the section is responsible for almost all of this transport. We expect that information about absolute sea-surface height will add a barotropic component to the flow and adjust the reference velocities at the bottom of the domain accordingly.

\section{Estimate from hydrography and sea-surface height data}

\subsection{Sea-surface height data}

For a mean sea-surface height, the mean seasurface CLS_SHOM98.2 by Hernandez and Schaeffer (2000) is used. This data product is a Gauss-Markov estimate of the mean elevation above a reference ellipsoid from satellite altimetry data of the GEOSAT-, TOPEX/POSEIDON-, and ERS-missions. In this sense, it is a temporal mean over the years 1986-1996, but as one of the reviewers pointed out to us, the TOPEX/POSEIDON data dominates the estimate so that CLS_SHOM98.2 is really a time mean over the years 1993-1996.

In order to obtain the dynamically relevant sea-surface elevation, an equipotential surface, the geoid, has to be subtracted from the CLS_SHOM98.2 data. We use the geoid height 
of the currently available geoid model EGM96 (Lemoine et al., 1997, the gridded geoid height values are available at http://cddisa.gsfc.nasa.gov/ 926/egm96/egm96.html). The EGM96 geoid model is a set of spherical harmonic coefficients up to degree and order 360. The geoid height was computed from the harmonic coefficients explicitly for the application with satellite altimetry, and the reference systems (reference ellipsoid, permanenttide system, etc.) are the same for both the mean sea-surface and the geoid height (Pavlis and Hernandez, pers. comm., 2003).

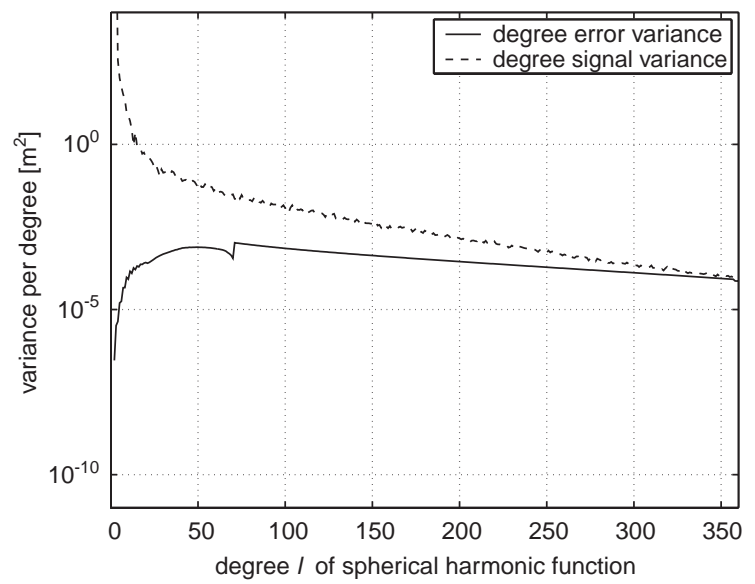

Fig. 3. Error variance and signal variance per degree for EGM96 (after Lemoine et al., 1997).
The geoid model coefficients were calculated by a variety of techniques. As a consequence, the model's error estimate is only complete to degree and order 70. Above degree 70 the error estimate was not computed from the full normal equations but is strictly diagonal. Because the errors below and above degree 70 are estimated by different techniques, there is a discontinuity in the error spectrum at degree 70 (Fig. 3). This degree corresponds to a resolution (half wavelength) of approximately $300 \mathrm{~km}$. The resolution of both the CLS_SHOM98.2 data and the inverse model's grid (order $50 \mathrm{~km}$ ) is much higher than that.

In Fig. 4(a), the altimetric sea-surface height SSH_GEO is shown together with the combined errors of altimetry and geoid, interpolated bilinearly from a $0.25^{\circ} \times 0.25^{\circ}$ grid (solid line and gray envelope). The altimeter errors are assumed to be of order $5 \mathrm{~cm}$, following Hernandez and Schaeffer (2000). The rough geoid error estimate is calculated from a geoid expansion to $180^{\circ}$, which roughly corresponds to a $1^{\circ}$ resolution. It is obvious from Fig. 4(a) that the large geoid errors dominate the total sea-surface height error. However, the blue geoid error spectrum (Lemoine et al., 1997, see also Fig. 3) and the discontinuity at degree 70 suggest using only the low-degree information for which the estimated errors are small.
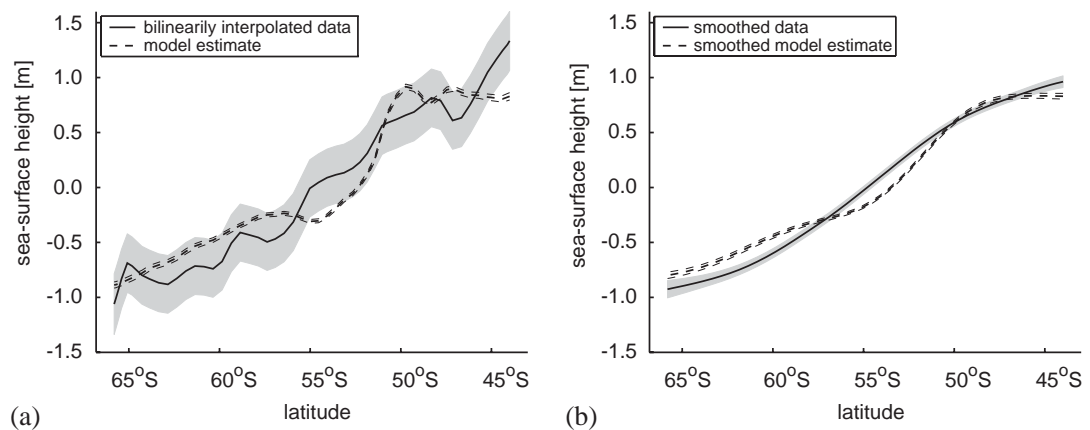

Fig. 4. A priori estimates of sea-surface height. All estimates have a zero mean. (a) Sea-surface height SSH_GEO that was bi-linearly interpolated from a $0.25^{\circ} \times 0.25^{\circ}$ grid without any further smoothing (solid line) with rough error estimate corresponding to an EGM96 expansion to degree 180 (gray envelope); sea-surface height estimate by the inverse model from the hydrographic measurements alone with a prior reference velocity error of $1 \mathrm{~cm} \mathrm{~s}^{-1}$ (SSH_HYD, thick dashed line); the thin dashed lines indicate the uncertainty of the model estimate. (b) Smoothed sea-surface height SSH_GEO with smoothed errors; smoothed estimate of the inverse model SSH_HYD. 
In order to do so, a filter is used here that has been derived especially for the purpose of removing all scales from the sea-surface height corresponding to a spherical harmonic degree higher than 70. It damps high degree (high wavenumber) spherical harmonics of both the signal and the errors effectively (Jekeli, 1981, details of the filter can also be found in Wahr et al., 1998, or Losch et al., 2002a.). Its characteristic shape is Gaussian both in spatial and in spectral space with a halfwidth of $286 \mathrm{~km}$. Data points outside of four times the half-width are neglected. The disadvantage of this filter is evident from Fig. 4(b): The surface elevation data in Fig. 4(a) has already little power below a wavelength of approximately $50 \mathrm{~km}$; a single application of the Gaussian filter smooths the data even further thereby removing part of the signal. But on the remaining scales the surface elevation data is much more accurate.

The error covariance for sea-surface height data (symbol $\mathbf{C}_{\mathrm{D}}$ in the appendix) is the sum of the geoid error covariance to degree and order 360 and the error covariance of the altimeter data. Both are provided with the data. Before smoothing, the magnitude of the two error contributions is on the order of 30 and $5 \mathrm{~cm}$, respectively. This sum has to be smoothed in the same manner as the data to describe the correct scales. Therefore, in the cost function, the difference between the sea-surface height data (SSH_GEO) and the model counterparts is weighted by a matrix that is constructed from the Gaussian filter and the data error covariance (see the appendix). The procedure both smoothes the sea-surface elevation data and the corresponding model estimate and thereby ensures that only the remaining long scales are adjusted in the inversion. Short scales of the sea-surface elevation data have effectively infinite errors and do not affect the model.

Losch et al. (2002b) showed that including the omission errors of the geoid model, which are due to finite resolution, increases the commission, that is, the resolved errors dramatically. A further reason for the use of the Gaussian filter is that it minimizes this aliasing effect. This is necessary, because a large prior error for the sea-surface height makes the data useless and precludes any improvement a priori (Ganachaud et al., 1997).

\subsection{Comparison to hydrographic estimate}

Fig. 4 compares the sea-surface height data to the model estimate that was obtained from hydrography alone. Within its errors of up to $28 \mathrm{~cm}$, the bi-linearly interpolated sea-surface height data (SSH_GEO, solid line) and the seasurface height estimate of the inverse model from the hydrographic measurements alone (SSH_HYD, thick dashed line, the thin dashed lines are the estimated errors) in Fig. 4(a) are generally consistent as in Ganachaud et al. (1997). After filtering the data to remove scales smaller than $286 \mathrm{~km}$ (corresponding to the EGM96 geoid resolution of degree 70), the errors, represented by the gray envelope around the solid line in Fig. 4(b), are much smaller. They barely reach $8 \mathrm{~cm}$ on scales longer than $286 \mathrm{~km}$. On these long scales, the altimetry data deviate from the filtered hydrographic estimate (dashed line) by more than the filtered errors. The overall drop of sea-surface height across the section according to the altimeter data is larger than that estimated from hydrography implying a larger total volume transport. We therefore conclude that our model estimate and the sea-surface height data are inconsistent on the (long) scales resolved by the EGM96.

At this stage one has to reject the prior assumptions made about the different errors involved. Either the errors of the altimetric height, the geoid height, or of the ocean model are underestimated. It may also be any combination or all three error sources that need to be revised.

Let us begin with the altimeter surface. Usually the error budget is known to a high accuracy (Chelton et al., 2000), and the error estimate of Hernandez and Schaeffer (2000) can be trusted. In addition, compared to the geoid the altimeter error budget remains a minor term and the total error is always dominated by the geoid error. In the next paragraph we will revise the ocean model's errors and increase the prior error for the bottom velocities to $5 \mathrm{~cm} \mathrm{~s}^{-1}$. We will then show that this large error is sufficient to remove the contradiction that was pointed out earlier before the different data from hydrography, geoid, and altimetry are combined. Unfortunately, the solution from this combination is not meaningful when compared 
with general knowledge about the Southern Ocean. We conclude that it is insufficient to revise altimeter and ocean model error. Additionally (or instead) the geoid error must be revised, i.e., it is larger than the estimates provided by the EGM96 authors.

Fig. 5 shows the long-wavelength comparison between SSH_GEO and SSH_HYD as Fig. 4(b) but for a revised prior error of $5 \mathrm{~cm} \mathrm{~s}^{-1}$ for the reference velocities. No altimeter data are assimilated yet. As a consequence of the larger prior error, the model's estimated errors increase by a factor of about five. The sea-surface height data errors and the model errors now overlap almost everywhere so that the new estimate can be considered to be consistent with the data. The new velocity field, which is shown in the middle panel of Fig. 2, can barely be distinguished by eye from the estimate with the smaller prior reference velocity errors (Fig. 2, top panel). However, the estimated transport errors all increase by a factor of about five as well and the new estimate of the total volume transport is $154 \pm 62 \mathrm{~Sv}$. While the error estimate is much larger than for mean transport estimates (Macdonald, 1998; Sloyan and Rintoul, 2000; Ganachaud and Wunsch, 2000; Rintoul and Sokolov, 2001), one can in principle interpret it as a consequence of the strong barotropic component of the ACC.

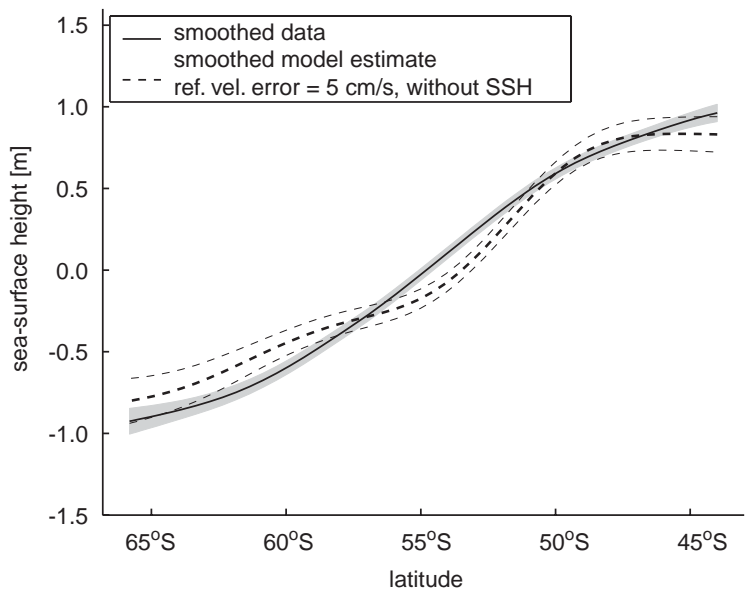

Fig. 5. Same as Fig. 4(b) with a prior error for the reference velocity of $5 \mathrm{~cm} \mathrm{~s}^{-1}$; sea-surface height data is not included in the inversion.

\subsection{Combining hydrography and sea-surface height data}

In the final step we combine the sea-surface height and the new, revised hydrographic estimate. Due to the increased error level, the inverse model is sufficiently flexible to easily adjust to reference velocities and fit the measured large scale seasurface height well, see Fig. 6. However, the new flow field estimate is very much distorted (Fig. 2, bottom panel). In our solution, we find a strong westward bottom current beneath the Subantarctic Front in the core of the ACC. This current has not been observed and contradicts the generally accepted notion of a deep-reaching ACC (Rintoul et al., 2001; Donohue et al., 2001). Also, the deep counter current reduces the transport in the Subantarctic Front from 115 to $85 \mathrm{~Sv}$, which contradicts estimates from combined current meter and hydrographic data by Phillips and Rintoul (2002). Donohue et al. (2001) find, admittedly much further to the East, that the geostrophic velocity referenced to the bottom generally underestimates the transports in the Subantarctic Front, when compared to estimates that include absolute velocity measurements from shipboard acoustic Doppler current profiler. In our case, in contrast, information about the

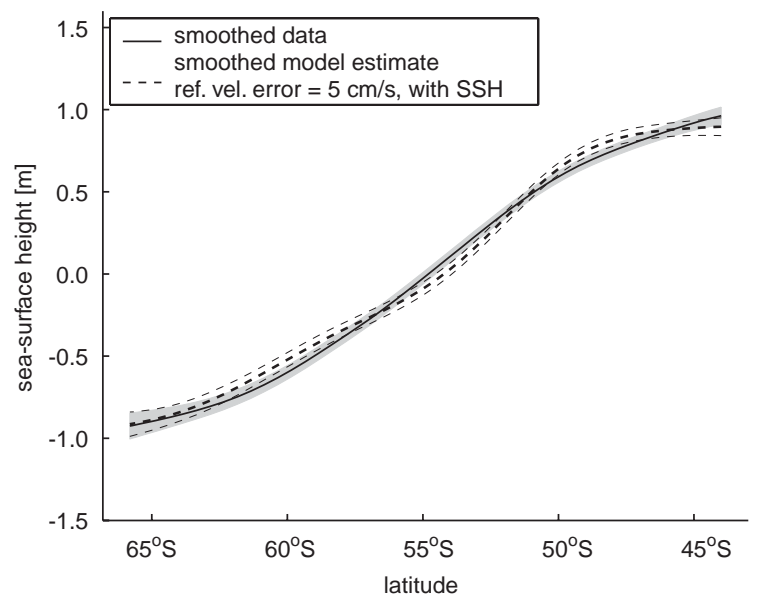

Fig. 6. Same as Fig. 5 with sea-surface height data included in the inversion (SSH_ALT); the prior error for the reference velocity is $5 \mathrm{~cm} \mathrm{~s}^{-1}$. 
absolute velocities reduces the transport of the Subantarctic Front substantially.

Even the recent result of Meinen and Luther (2003) suggests from additional measurements with inverted echo sounders and horizontal electric field recorders, that there is no deep westward current in the Subantarctic Front. This is further evidence to support the conclusion that our estimate with sea-surface height is not consistent with prior oceanographic knowledge about the Subantarctic Front.

Further, the strength of the drift current south of $55^{\circ} \mathrm{S}$ has been dramatically increased with seasurface height data. As a consequence, the total estimated transport in this experiment of $210 \pm 27 \mathrm{~Sv}$, while still formally consistent with our second estimate without sea-surface height data, is far greater than any previous estimate derived from measurements (Yaremchuk et al., 2001; Rintoul et al., 2001; Rintoul and Sokolov, 2001). Also, the actual flow field in the bottom panel Fig. 2 with large velocities near the bottom appears to be unrealistic which leads us to conclude that with sea-surface height data the net transport is overestimated.

The hydrographic measurements are given a small weight in the cost function to account for noise due to ocean variability. Additionally, the weighting scheme allows for large mutual variations of temperature and salinity that are consistent with vertical correlations that were estimated a priori. These variations could lead to vertical displacements of density surfaces in the model estimate. Still the model adjusts to the sea-surface height data mainly by shifts in the reference velocity; the density structure along the section is hardly affected by the sea-surface height data and remains close to what has been estimated without them (not shown, see Yaremchuk et al., 2001, for a further discussion). We emphasize that, due to our weighting scheme, only the long scale components, which are resolved by the filtered sea-surface height data, are adjusted in the model and that the short scale structure of the velocity field of the hydrography-only solution remains almost unchanged.

Note that because of our choice of large prior error estimates for the reference velocities of
$5 \mathrm{~cm} \mathrm{~s}^{-1}$, the total transport error can be reduced from 62 to $27 \mathrm{~Sv}$ by including altimetry in the solution. This result is different to that of Ganachaud et al. (1997) who found that, with larger prior data errors implied by the older, less accurate geoid model JGM-3, the impact of seasurface height data is negligible. Here, we report a strong impact with the more accurate EGM96. However, the impact did not improve the solution. Instead the flow field is more realistic without satellite data.

\section{Discussion}

The inconsistent transport estimate and the unrealistic velocity field obtained with the seasurface height data imply that the smoothed seasurface height relative to the EGM96 geoid height overestimates the sea-surface drop across the Southern Ocean and consequently overestimates the total transport as well. In this context, the formal error estimate of the EGM96 geoid height, which dominates the sea-surface height errors, appears to be too small even on the long, presumably well-known, scales. Therefore, the inverse model produces a total transport that is too large when model and data are made consistent by substantially increasing the model uncertainties prior to inversion. This leads us to the conclusion that sea-surface height data relative to the EGM96 is not compatible with our present understanding of the ACC and its structure south of Australia. Most likely the formal errors of EGM96 underestimate the true errors and need to be revised.

LeGrand et al. (2003) came to a different conclusion. With their inverse model, they were able to combine a mean sea-surface topography, derived from TOPEX/POSEIDON satellite data and the EGM96, with data from a hydrographic atlas to give a new estimate of the mean sea surface topography. This new estimate "appears to be qualitatively consistent with previous oceanographic knowledge" (LeGrand et al., 2003). LeGrand et al. (2003) used data that is heavily smoothed. For example, the sea-surface data were smoothed with an averaging radius of the order of $400 \mathrm{~m}$, which corresponds approximately to a 
spherical harmonic degree of $50(\approx \pi$ [Radius of Earth] $/ 400 \mathrm{~km}$ ); note that we use a Gaussian filter with a roll-off that corresponds to degree 70 . But the errors that LeGrand et al. used describe degrees up to 180 , which corresponds to scales down to almost $100 \mathrm{~km}$. No further smoothing of the errors is described and we must assume that the unsmoothed error estimate was used. The cumulative error variance (square of the errors) to degree 180 is globally larger than the error variance to degree 50 by approximately $(27 \mathrm{~cm})^{2}$ or a factor of 4 . In fact, if one attaches the error estimate to degree 180 in Fig. 4(a) to the smoothed data (approximately to degree 70) in Fig. 4(b), smoothed data and model are immediately compatible. It is with their (legitimate) choice of the large prior error estimate for the sea surface topography data, that LeGrand et al. (2003) find a solution that is consistent with "previous oceanographic knowledge." We suspect that if they used errors that describe only the scales that are resolved in the data, their result would confirm our conclusion, namely that the formal error estimates of EGM96 are too small.

Implications of inconsistencies between ocean models and geoid models that emerge when the modeled sea-surface height is compared to data are not new. But we can-to our knowledge for the first time - show in an analysis complete with formal error estimates that hydrography and seasurface height relative to EGM96 cannot be consistently combined in the framework of geostrophic dynamics. An increased geoid error could remove this inconsistency. Ganachaud et al. (1997) have shown that with the JGM-3, which has errors that are approximately twice as large as the EGM96 errors, the altimetric and the hydrographic information are consistent. But they also found that, because of the large geoid errors, the altimeter data was nearly useless in improving the solution error estimates, except for a small impact in the Southern Ocean. Therefore, before using absolute sea-surface height data in combination with geoid models and ocean models, it is absolutely necessary to decrease (or at least retain) the current formal error levels of the geoid models and at the same time reduce the true errors of these models. Ongoing and future space-borne gravity missions, for example the Gravity Recovery and Climate Experiment (GRACE Tapley, 1997) and Gravity Field and Steady-State Ocean Circulation Explorer (GOCE Battrick, 1999), have this objective.

The particular hydrographic section we have studied lends itself naturally to the use of altimetry. The flow through this section determines the transport around two continents, Australia and Antarctica. These transports are important to all of the Southern Ocean. At the same time, they are poorly measured. Furthermore, the width of the Southern Ocean south of Australia makes altimetry an attractive technique because the accuracy of geoid height data is highest on the large scales. It is remarkable that even in these favorable circumstances proper use of the data leads to inconsistent results. From our investigation, we are forced to conclude that until improvements in geoid modeling are achieved, ocean state estimation should be performed without reference to an absolute sea-surface height. But note the following caveat: we have only studied one hydrographic section. Therefore, extrapolating this conclusion globally bears a risk, and more studies of this type in different regions of the world are necessary to confirm our findings.

\section{Acknowledgements}

The authors thank Alexey Yaremchuk for his help with the error computations. We acknowledge the valuable contribution of Ralf Giering's Tangent linear Adjoint Model Compiler (Giering and Kaminski, 1998) in speeding the production of the adjoint code to the model. Francis Condi and Arnaud Czaja read early versions of the manuscript and made valuable suggestions. Discussions with Victor Zlotnicki, Nikos Pavlis, Fabrice Hernandez, and Pascal LeGrand greatly improved the manuscript.

\section{Appendix A. Construction of the weight matrix for the sea-surface height data}

The term in the cost function that minimizes the difference between sea-surface data $\boldsymbol{\eta}_{\mathrm{D}}$ and 
corresponding model estimate $\boldsymbol{\eta}$ ideally has the form

$$
\left(\mathbf{H} \boldsymbol{\eta}-\boldsymbol{\eta}_{\mathrm{D}}\right)^{\mathrm{T}} \mathbf{C}_{\mathrm{D}}^{-1}\left(\mathbf{H} \eta-\eta_{\mathrm{D}}\right),
$$

where $\mathbf{H}$ is the interpolation (or observation) operator that maps the model sea-surface height onto the data locations (Thacker, 1989). In state estimation the model-data misfit is weighted by the inverse of the data error covariance matrix $\mathbf{C}_{D}$. Since, in our case, the data is available on a global grid, but the model grid is in effect only a line of grid points, finding $\mathbf{H}$ is not straightforward. It could be constructed as a pseudo-inverse of the Gaussian smoothing operator $\mathbf{Q}$ that maps the data values onto the model grid

$$
\mathbf{Q}: \boldsymbol{\eta}_{\mathrm{D}} \mapsto \mathbf{Q} \boldsymbol{\eta}_{\mathrm{D}}=\mathbf{V S U}^{\mathrm{T}} \mathbf{C}_{\mathrm{D}}^{-1} \boldsymbol{\eta}_{\mathrm{D}}
$$

In the singular value decomposition of $\mathbf{Q}, \mathbf{U}$ is the matrix of basis vectors of the data grid (or space), $\mathbf{V}$ the matrix of basis vectors of the model grid (or space), and $\mathbf{S}$ the diagonal matrix of singular values. The inverse of the data error covariance matrix $\mathbf{C}_{\mathrm{D}}$ is a natural choice for the metric on the data grid and the basis vectors $\mathbf{U}$ are chosen such that $\mathbf{U}^{\mathrm{T}} \mathbf{C}_{\mathrm{D}}^{-1} \mathbf{U}=\mathbf{1}$, that is $\mathbf{U}$ contains the units. On the model grid, $\mathbf{V}^{\mathrm{T}} \mathbf{V}=\mathbf{1}$. The pseudo-inverse of $\mathbf{Q}$ can then be found by inverting (A.2)

$$
\mathbf{H}: \boldsymbol{\eta} \mapsto \mathbf{H} \boldsymbol{\eta}=\mathbf{U S}^{-1} \mathbf{V}^{\mathrm{T}} \boldsymbol{\eta} .
$$

In practice, $\mathbf{S}$ contains some very small singular values that would amplify noise in $\boldsymbol{\eta}$. In order to suppress that noise, the inverse of $\mathbf{S}$ can be replaced by a matrix $\tilde{\mathbf{S}}^{-1}$ in which the inverses of the small singular values have been set to zero. In this way only those structures of the model estimate are retained that can be resolved on the data grid.

But instead of constructing an interpolation operator $\mathbf{H}$, we let Eqs. (A.2) and (A.3) motivate a different cost function term for the sea-surface height

$\left(\boldsymbol{\eta}-\mathbf{Q} \boldsymbol{\eta}_{\mathrm{D}}\right)^{\mathrm{T}} \mathbf{W}\left(\boldsymbol{\eta}-\mathbf{Q} \boldsymbol{\eta}_{\mathrm{D}}\right)$.

Again, the weight matrix $\mathbf{W}$ should be the inverse of the data error covariance matrix restricted to the section coordinate: $\left(\mathbf{Q} \mathbf{C}_{\mathrm{D}} \mathbf{Q}^{\mathrm{T}}\right)^{-1}$. In general, this inverse does not exist if the data resolution is lower than the grid resolution of the model. Instead, the weight matrix is constructed by using

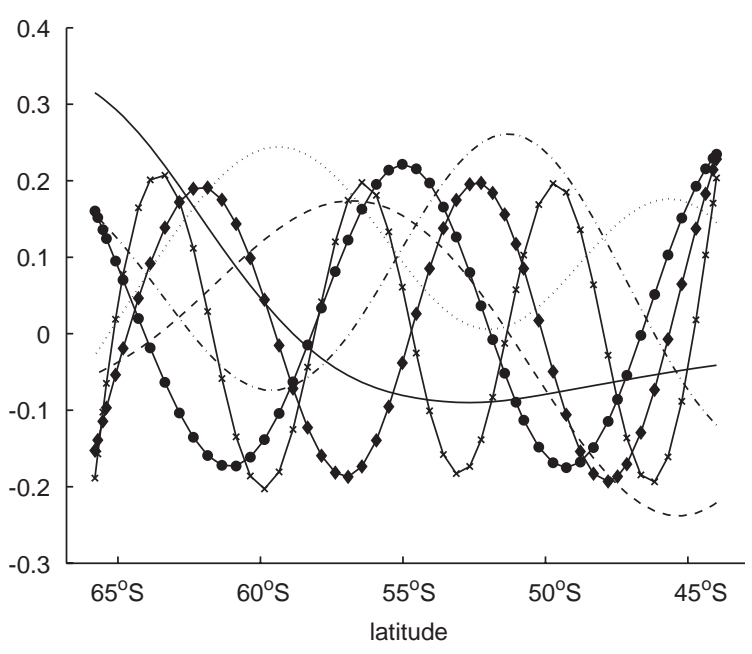

Fig. 7. Singular vectors $\mathbf{V}$ from which the sea-surface height weight matrix is constructed.

(A.2) with $\mathbf{S}$ replaced by $\tilde{\mathbf{S}}$, so that

$\mathbf{W}=\mathbf{V} \tilde{\mathbf{S}}^{-2} \mathbf{V}^{\mathrm{T}}$.

It is immediately clear that with cost function (A.4) only the model coefficients $\mathbf{V}^{\mathrm{T}} \boldsymbol{\eta}$ that correspond to non-zero singular values are fitted to the data coefficients $\tilde{\mathbf{S}} \mathbf{U}^{\mathrm{T}} \mathbf{C}_{\mathrm{D}}^{-1} \boldsymbol{\eta}_{\mathrm{D}}$, weighted by the inverse of the singular values $\tilde{\mathbf{S}}^{2}$.

The art of this procedure is selecting the criteria for the smallness of the singular values of $\mathbf{S}$. We set those singular values $s_{k}$ to zero for which $\left\|s_{k}^{-1} \mathbf{u}_{k}\right\|_{\infty}>2$. The remaining 7 singular values constitute $\tilde{\mathbf{S}}$. The corresponding vectors $\mathbf{V}$ are plotted in Fig. 7. Then the expression (A.4) corresponds to an interpolation operator $\mathbf{H}$ in cost function (A.1) that filters out components of the model estimate that are not resolvable on the data grid. The filtered-out components do not contribute to the cost function and they are totally unconstrained by the satellite observations.

\section{References}

Battrick, B. (Ed.), 1999. Gravity Field and Steady-State Ocean Circulation Explorer. ESA SP-1233 (1)-The Four Candidate Earth Explorer Core Missions. ESA, c/o ESTEC, Noordwijk, The Netherlands.

Chelton, D.B., Ries, J.C., Haines, B.J., Fu, L.-L., Callahan, P.S., 2000. Satellite altimetry. In: Fu, L.-L., Cazenave, A. 
(Eds.), Satellite Altimetry and Earth Sciences, A Handbook of Techniques and Applications. International Geophysics Series, Vol. 69, Academic Press, San Diego, pp. 1-131.

Donohue, K.A., Firing, E., Chen, S., 2001. Absolute geostrophic velocity within the Subantarctic Front in the Pacific Ocean. J. Geophys. Res. 106 (C9), 19869-19882.

Ganachaud, A., Wunsch, C., 2000. Improved estimates of global ocean circulation, heat transport and mixing from hydrographic data. Nature 408, 453-457.

Ganachaud, A., Wunsch, C., Kim, M.-C., Tapley, B., 1997. Combination of TOPEX/POSEIDON data with a hydrographic inversion for determination of the oceanic general circulation and its relation to geoid accuracy. Geophys. J. Int. 128, 708-722.

Giering, R., Kaminski, T., 1998. Recipies for adjoint code construction. ACM Trans. Math. Software 24 (4), 437-474.

Gouretski, V.V., Jancke, K., 1998. WOCE Report No. 162/98. Technical Report 3, WHP Special Analysis Center; http:// www.dkrz.de/ u241046/SACserver/SACHome.htm.

Hernandez, F., Schaeffer, P., 2000. Altimetric mean sea surfaces and gravity anomaly maps inter-comparisons. AVISO Technical Report AVI-NT-011-5242-CLS, CLS, Toulouse, France.

Jekeli, C., 1981. Alternative methods to smooth the Earth's gravity field. Technical Report 327, Department of Geodetic Science and Surveying, Ohio State University.

LeGrand, P., 2001. Impact of the Gravity Field and SteadyState Ocean Circulation Explorer (GOCE) mission on ocean circulation estimates: volume fluxes in the climatological inverse model of the Atlantic. J. Geophys. Res. 106 (C9), 19597-19610.

LeGrand, P., Schrama, E.J.O., Tournadre, J., 2003. An inverse estimate of the dynamic topography of the ocean. Geophys. Res. Lett. 30 (2), 1062 (doi:10.1029/2002GL014917).

Lemoine, F.G., Smith, D.E., Kunz, L., Smith, R., Pavlis, E.C., Pavlis, N.K., Klosko, S.M., Chinn, D.S., Torrence, M.H., Williamson, R.G., Cox, C.M., Rachlin, K.E., Wang, Y., Kenyon, S.C., Salman, R., Trimmer, R., Rapp, R.H., Nerem, R.S., 1997. The development of the NASA GSFC and NIMA joint geopotential model. In: Segawa, J., Fujimoto, H., Okubo, S. (Eds.), Gravity, Geoid and Marine Geodesy, Vol. 117. International Association of Geodesy Symposia, Springer, New York, pp. 461-469.

Losch, M., Redler, R., Schröter, J., 2002a. Estimating a mean ocean state from hydrography and sea-surface height data with a non-linear inverse section model: Twin experiments with a synthetic data set. J. Phys. Oceanogr. 32 (7), 20962112.

Losch, M., Sloyan, B.M., Schröter, J., Sneeuw, N., 2002b. Box inverse models, altimetry and the geoid: problems with the omission error. J. Geophys. Res. 107 (C7) 10.1029/ $2001 \mathrm{JC} 000855$.

Macdonald, A.M., 1998. The global ocean circulation: a hydrographic estimate and regional analysis. Prog. Oceanogr. 41, 281-382.

Martel, F., Wunsch, C., 1993. Combined inversion of hydrography, current meter data and altimetric elevations for the North Atlantic circulation. Manuscr. Geodaet. 18, 219-266.
Meinen, C.S., Luther, D.S., 2003. Comparison of methods of estimating mean synoptic current structure in "stream coordinates" reference frames with an example from the Antarctic circumpolar current. Deep-Sea Res. 50 (2), 201-220.

Nechaev, D.A., Yaremchuk, M.I., 1995. Application of the adjoint technique to processing of a standard section data set: World Ocean Circulation Experiment section S4 along $67^{\circ} \mathrm{S}$ in the Pacific Ocean. J. Geophys. Res. 100 (C1), 865-879.

Phillips, H.E., Rintoul, S.R., 2002. A mean synoptic view of the Subantarctic Front south of Australia. J. Phys. Oceanogr. 32 (5), 1536-1553.

Rapp, R.H., Zhang, C., Yi, Y., 1996. Analysis of dynamic ocean topography using TOPEX data and orthonormal functions. J. Geophys. Res. 101 (C10), 22583-22598.

Rintoul, S.R., Sokolov, S., 2001. Baroclinic transport variability of the Antarctic Circumpolar Current south of Australia (WOCE repeat section SR3). J. Geophys. Res. 106 (C2), 2795-2814.

Rintoul, S.R., Hughes, C., Olbers, D., 2001. The Antarctic circumpolar current system. In: Siedler, G., Church, J., Gould, J. (Eds.), Ocean Circulation and Climate, International Geophysics Series, Vol. 77, Academic Press, New York, pp. 271-302.

Rosenberg, M., Eriksen, R., Bell, S., Bindoff, N., Rintoul, S., 1995. Aurora Australis marine science cruise AU9407oceanographic field measurements and analysis. Antarctic CRC Research Report 6, Antarctic CRC, Hobart, Australia.

Schröter, J., Losch, M., Sloyan, B.M., 2002. Impact of the Gravity Field and Steady-State Ocean Circulation Explorer (GOCE) mission on ocean circulation estimates: volume and heat transports across hydrographic sections. J. Geophys. Res. 107 (C2) (doi:10.1029/ 2000JC000647).

Sloyan, B.M., Rintoul, S.R., 2000. Estimates of area-averaged diapycnal fluxes from basin-scale budgets, J. Phys. Oceanogr. 2320-2341.

Stammer, D., Wunsch, C., 1994. Preliminary assessment of the accuracy and precision of TOPEX/POSEIDON altimeter data with respect to the large-scale circulation. J. Geophys. Res. 99 (C12), 24584-24604.

Stammer, D., Wunsch, C., Giering, R., Eckert, C., Heimbach, P., Marotzke, J., Adcroft, A., Hill, C.N., Marshall, J., 2002. The global ocean circulation during 1992-1997, estimated from ocean observations and a general circulation model. J. Geophys. Res. 107 (C9), 3118 (doi:10.1029/ 2001JC000888).

Tapley, B.D., 1997. The gravity recovery and climate experiment (GRACE). Suppl. Trans. Am. Geophys. Union (EOS) 78 (46), 163.

Thacker, W.C., 1989. On the role of the Hessian matrix in fitting models to data. J. Geophys. Res. 94 (C5), 6177-6196.

Wahr, J., Molenaar, M., Bryan, F., 1998. Time variability of the Earth's gravity field: Hydrological and oceanic effects 
and their possible detection using GRACE. J. Geophys. Res. 103 (B12), 30205-30299.

Wenzel, M., Schröter, J., Olbers, D., 2001. The annual cycle of the global ocean circulation as determined by 4D VAR data assimilation. Prog. Oceanogr. 48, 73-119.

Wunsch, C., 1978. The North Atlantic general circulation west of $50^{\circ}$ determined by inverse methods. Rev. Geophys. Space Phys. 16, 583-620.

Wunsch, C., 1993. Physics of the ocean circulation. in: Rummel, R., Sansò, F. (Eds.), Satellite Altimetry in Geodesy and
Oceanography, Lecture Notes in Earth Sciences, Vol. 50, Springer, Berlin, pp. 9-98.

Wunsch, C., Stammer, D., 1998. Satellite altimetry, the marine geoid, and the oceanic general circulation. Ann. Rev. Earth and Planet. Sci. 26, 219-253.

Yaremchuk, M.I., Bindoff, N.L., Schröter, J., Nechaev, D., Rintoul, S.R., 2001. On the zonal and meridional circulation and ocean transports between Tasmania and Antarctica. J. Geophys. Res. 106 (C2), 2815-2832. 